\title{
A case of pemphigus foliaceus and pustular psoriasis with a brief review of literature
}

\author{
Darjani Abbas', Rafiei Rana1, Mesbah Alireza², Shafaei Sareh', Rafiee Behnam³ \\ ${ }^{\text {}}$ Skin Research Center, Guilan University of Medical Sciences, Razi Hospital, SardareJangal Street, Rasht, Iran, \\ ${ }^{2}$ Razi Pathobiology, Rasht, Iran, ${ }^{3}$ Research Intern. University of Texas, MD Anderson Cancer Center 1515 Holcombe blvd. \\ B4.4512, Houston, TX, 77030, USA
}

Corresponding author: Rafiei Rana, MD, E-mail: rafieirana@yahoo.com

\begin{abstract}
Rarely pemphigus foliaceus could be associated with generalized pustular psoriasis. A 66-year-old woman with diffuse flaccid bullae and erosions of pemphigus foliaceus underwent two sessions of pulse therapy of corticosteroid and oral prednisolone during the interval time. Two weeks after the second session of pulse therapy with improvement of the lesions, during tapered dosage of oral prednisolone, facial annular erythematous lesions appeared and were superimposed by generalized pustular eruptions. Skin biopsy of pustular lesions showed pustular psoriasis so intramuscular methotrexate was added. Two years later, during decreasing dosage of methotrexate and prednisolone, she had eruptive recurrence of pustules and flaccid bullae associated with erythroderma and fever which were controlled with increasing dosage of corticosteroid and starting oral retinoid. These hypotheses may explain co-occurring pemphigus foliaceus and pustular psoriasis: decreasing dosage of corticosteroid, interleukin-8 overproduction in keratinocytes and increased activity of plasminogen activator in skin lesions.
\end{abstract}

Key words: Pemphigus foliaceus; pustular psoriasis; immunobullous disease

\section{INTRODUCTION}

Pemphigus foliaceus (PF) is an autoimmune bullous disease (AIBD) which is presented by superficial flaccid blisters, erosions, scales and crusts on the seborrheic areas of the face and trunk without mucosal involvement [1]. Pathologically, PF is characterized by granular layer acantholysis with acantholytic cells and intraepidermal deposition of Immunoglobulin $\mathrm{G}(\mathrm{IgG})$ and complement component 3 (C3) on immunofluorescent studies as a result of antibody production against desmoglein 1, a component of desmosome [2]. Although psoriasis and AIBD are classified as different diseases, PF coexistent with psoriasis vulgaris has been reported in a few cases [3], but association with pustular psoriasis has been very rare $[4,5]$.

Micro-environmental factors could be able to induce concurrent autoimmune phenomenon in an individual patient [3]. Herein, we report a case of PF and generalized pustular psoriasis (GPP).

\section{CASE REPORT}

\section{First Admission}

In 2014, a 64-year-old woman with diffuse flaccid superficial bullae and erosions from 2 months ago was admitted to our hospital. In physical examination there were extensive bullae, erosions, scales and crusts on the scalp, face, trunk and limbs with no typical pre-existing psoriasis lesions. Nikolsky sign was positive. Mucosal surfaces were intact. Onychomycosis in toe nails with no pitting or oil spot was detected. In past medical history she had a hysterectomy 20 years ago and hyperlipidemia which was under control with atorvastatin $(20 \mathrm{mg}$ daily). There was no history of psoriasis or bullous diseases in her family.

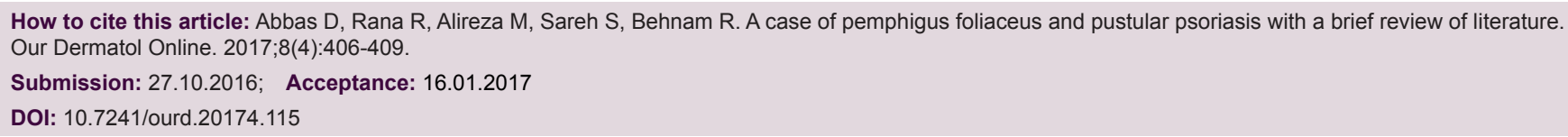


Biochemical and hematologic laboratory evaluations were within normal limits. Biopsy of the bullous lesion and perilesional area was made. Biopsy samples showed subcorneal cleft with acantholytic cells and intercellular deposition of IgG and C3 (Fig. la-c) in direct immunofluorescent (DIF) studies which confirmed the diagnosis of PF. Indirect immunofluorescent (IIF) studies were not performed.

According to the extensity and severity of her disease, two sessions of pulse therapy with corticosteroid (1 gr intravenous methyl prednisolone daily for three consecutive days) with one month interval and oral prednisolone $60 \mathrm{mg}$ daily in the interval time were administered. Bullous lesions were controlled after one month, so oral prednisolone dosage was decreased to $50 \mathrm{mg}$ daily but after two weeks, facial annular erythematous lesions (Fig. 2a) appeared which were followed by generalized pustular eruptions associated with fever, burning and pruritus. Tzanck smear, potassium hydroxide examination and microbial culture of new lesions were non-remarkable. In new laboratory evaluations there were elevated erythrocyte sedimentation rate (ESR: 67) and leukocytosis with neutrophilia. Serum levels of calcium, zinc, albumin and antinuclear antibody (ANA) were within normal limits. Blood and urine cultures were negative. Again biopsy was made from new pustular lesions which showed mild spongiosis with subcorneal neutrophilic pustules without acantholytic cells or eosinophilic infiltration (Fig. 3a and b). DIF studies of perilesional area of new pustules were non-remarkable, therefore diagnosis of generalized pustular psoriasis (GPP) was proposed and intramuscular methotrexate (MTX) (12.5mg weekly) with oral folic acid (1 mg six days per week) were added to corticosteroid therapy. After two weeks pustular lesions were controlled so she was discharged from the hospital, her medications were tapered and she was followed in the outpatient clinic for two years. She had gradually become chushingoid (facial puffiness and weight gain), hypertensive and depressed, so hydrochlorothiazide, captopril, nitrocontin and alprazolam were added to her medications.

\section{Second Admission}

Two years later in 2016, about two weeks after changing intramuscular MTX (5mg per week) to oral MTX (7.5mg per week) and tapering oral prednisolone to $10 \mathrm{mg}$ daily; she had recurrence of pustules, flaccid bullae, crusted and exudative lesions (Fig. 2b and c) which

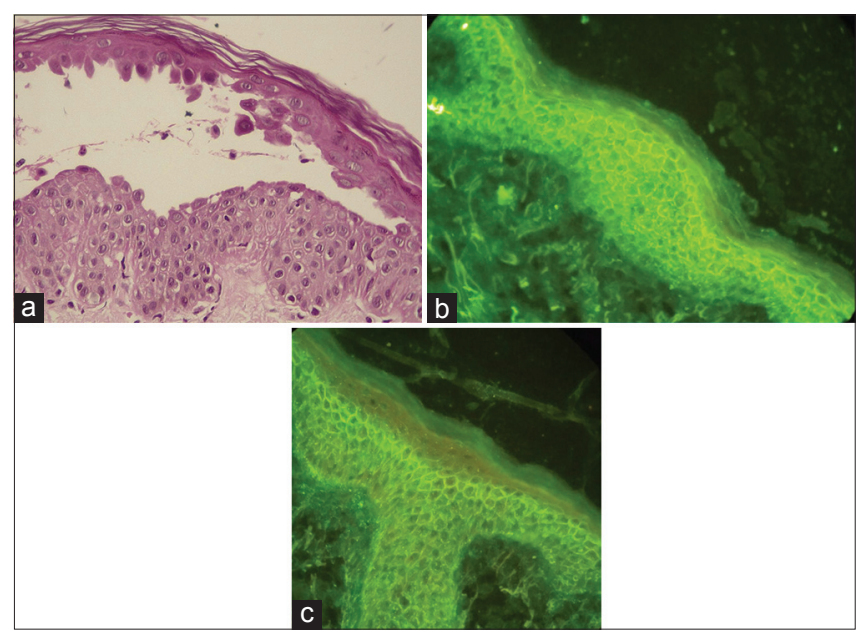

Figure 1: (a) Acantholysis of the upper epidermis with acantholytic cells [Hematoxylin-eosin, original magnification $\times 400$ ]; (b and c) Direct Immunofluorescent staining on the perilesional tissue demonstrated intercellular IgG and C3 depositions. (Original magnification $\times 200$ ).

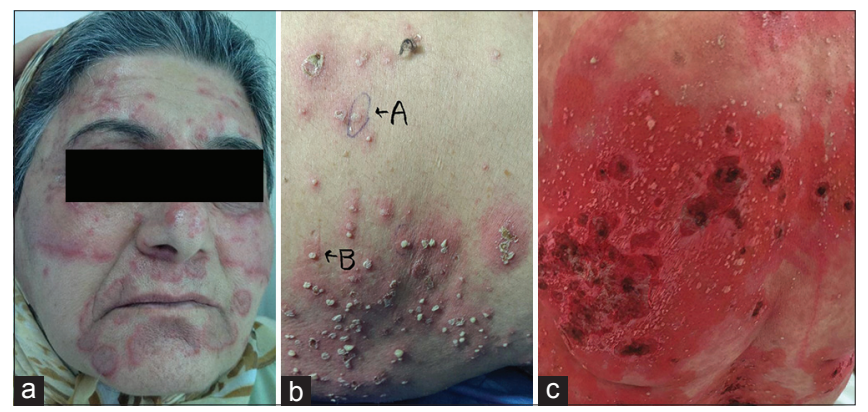

Figure 2: (a) Facial annular erythematous lesions before pustular eruptions. (b) Disseminated bullous (arrow A) and pustular lesions (arrow B) on the back. (c) Crusted lesions on the back which have been stained with eosin.

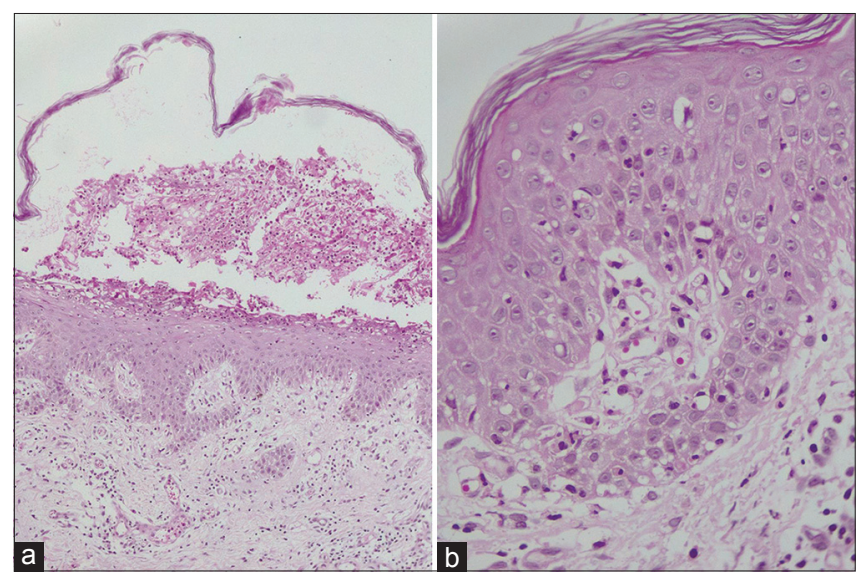

Figure 3: a) Subcorneal pustules; b) Transmigration of the neutrophils associated with dermal telangiectasia. (H\&E, original magnification: (a) $\times 100$, (b) $\times 400$ ).

were followed by erythroderma, fever and malaise, so she was admitted to the hospital again. In new laboratory evaluations, significant findings were: anemia (Hemoglobin: 11, Mean corpuscular volume: 87) with 
normal iron profile), leukocytosis with neutrophilia, raised ESR (ESR: 42), positive C-reactive protein (CRP: $3+$ ), hypoalbuminemia with total serum calcium concentration of $7 \mathrm{mg} / \mathrm{dl}$, leukocyturia with nitrite positivity in urine analysis.

Culture of exudative lesions revealed Methicillin-resistant Staphylococcus aureus, but urine and blood culture were negative. Serum levels of sodium, potassium, zinc and magnesium were within normal limits. Imaging findings (chest X-ray and abdominopelvic ultrasound) were no remarkable.

Parenteral hydrocortisone (150mg daily) and antibiotics (ceftazidim 2gr daily + clindamycin 1800 mg daily) were administered. Supportive cares for erythroderma and fever were done and MTX was discontinued. Her lesions were controlled after two weeks and oral prednisolone $50 \mathrm{mg}$ with oral retinoid (acitretin $25 \mathrm{mg}$ daily) was administered.

\section{DISCUSSION}

Our case initially had only bullous lesions which clinically and pathologically were diagnosed as PF but after decreasing the dosage of corticosteroid, she showed annular erythema and pustules with systemic symptoms, so the most probable diagnosis was PF superimposed by GPP.

Although rarely PF could be associated with GPP; interestingly we found case reports that PF has initially manifested as neutrophilic pustules but in those cases, pathologic changes and DIF studies of the pustules confirmed PF not GPP $[1,5,6]$. In our patient pustular lesions had no pathologic criteria of PF and were preceded by annular erythema, so she was not a case of pustular PF.

Psoriasis could be associated with AIBD especially bullous pemphigoid but coexistence of psoriasis with $\mathrm{PF}$ has been reported rarely. Patients with pustular psoriasis have been more prone to develop AIBD than other types of psoriasis. The onset of pustular psoriasis has been simultaneous, before or after the onset of AIBD [7]. Coincidence of PF and annular pustular psoriasis has been reported only in a few cases [4,5]. Our patient initially had annular lesions on the face which were superimposed by generalized eruptive pustular lesions with systemic symptoms, so we considered the diagnosis of PF and GPP.
This association has been explained by multiple hypotheses including: Common pathogenic pathway, common human leukocyte antigen (HLA) type in either diseases, treatment complications or just a simple coincidence [3,7-9].

Most authors have believed that it is more than a coincidence. We have found following comments about these co-occurrences in the literature:

- There has been significant decreased suppressor activity in psoriasis disease which results in increasing production of autoantibodies against skin antigens [9]

- Plasminogen activator which has a major role in the induction of acantholysis has been increased in psoriatic lesions $[8,9]$

- HLA-DR4 haplotype is a common HLA type in both diseases [8]

- IgG autoantibodies could be able to induce Interleukin-8 (IL8) expression in keratinocytes; IL-8 has an important role in the production of neutrophilic pustules [4]

- Corticosteroids which have been used for treatment of AIBD could be a trigger for pustular psoriasis [7] on the other hand phototherapy for psoriasis may be a trigger to produce endogenous pemphigus autoantibodies $[3,10]$; in addition heat and ultra violet (UV) radiation are exacerbating factors in PF [2]. Also interleukin-l (IL-1) is a proinflammatory cytokine which has a major role in AIBD, psoriasis and UV damage [7]

- Enalapril or penicillamin intake, topical dithranol or salicylic acid, transient hypo zincemia and potential infectious foci, all have been incriminated in previous case reports $[5,11]$.

We think that in our case, probable triggering factors for GPP include: tapered dosage of the corticosteroid, unknown transient electrolyte imbalance during pulse therapy with corticosteroid, captopril intake and skin infection.

In previous case reports corticosteroids plus ciclosporin, dapsone, MTX or acitretin have been used with moderate improvement and some recurrences during dosage tapering $[2,4,5]$.

In conclusion; although PF and GPP are considered as completely separate diseases, this case and similar cases confirm that there is a pathogenetic linkage in these autoimmune diseases. 


\section{Abbreviations}

Autoimmune bullous disease: (AIBD)

Pemphigus foliaceus: (PF)

Generalized pustular psoriasis: (GPP)

Direct immunofluorescent: (DIF)

Indirect immunofluorescent: (IIF)

Antinuclear antibody: (ANA)

Methotrexate: (MTX)

Human leukocyte antigen: (HLA)

Interleukin-8: (IL8)

Ultra violet: (UV)

Interleukin-1: (IL-1).

\section{REFERENCES}

1. Miyakura T, Yamamoto T, Okubo Y, Ishii N, Oyama B, Hashimoto $\mathrm{T}$, et al. Pemphigus foliaceus with prominent neutrophilic pustules initially presenting as erythroderma. Clin Exp Dermatol. 2009;34:e46-9.

2. Aghassi D, Dover JS. Pemphigus foliaceus induced by psoralen-UV-A. Arch Dermatol. 1998;134:1300-1.

3. Kwon HH, Kwon IH, Chung JH, Youn JI. Pemphigus Foliaceus Associated with Psoriasis during the Course of Narrow-Band UVB Therapy: A Simple Coincidence? Ann
Dermatol. 2011;23(Suppl 3):S281-S284.

4. Kato K, Hanafusa T, Igawa K, Tatsumi M, Takahashi Y, Yamanaka T, et al. A rare case of annular pustular psoriasis associated with pemphigus foliaceus. Ann Dermatol. 2014;26:260-1.

5. Claus S, Ziemer M, Simon JC, Treudler R. Coincidence of annular pustular psoriasis, pemphigus foliaceus, and leukocytoclastic vasculitis associated with chronic cholecystitis. J Dtsch Dermatol Ges. 2016;14:830-1.

6. Matsuo K, Komai A, Ishii K, Futei Y, Amagai M, Deguchi H, et al. Pemphigus foliaceus with prominent neutrophilic pustules. $\mathrm{Br} \mathrm{J}$ Dermatol. 2001;145:132-6.

7. Ohata C, Ishii N, Koga H, Fukuda S, Tateishi C, Tsuruta D, et al. Coexistence of autoimmune bullous diseases (AIBDs) and psoriasis: A series of 145 cases. J Am Acad Dermatol. 2015;73:50-5.

8. Perez GL, Agger WA, Abellera RM, Dahlberg P. Pemphigus foliaceus coexisting with IgA nephropathy in a patient with psoriasis vulgaris. Int J Dermatol. 1995;34:794-6.

9. Yokoo M, Oka D, Ueki H. Coexistence of psoriasis vulgaris and pemphigus foliaceus. Dermatologica. 1989;179:222-3.

10. Lee CW, Ro YS. Pemphigus developed on preexisting dermatoses. J Dermatol. 1994;21:213-5.

11. Lee CW, Ro YS, Kim JH, Kim JH. Concurrent development of pemphigus foliaceus and psoriasis. Int J Dermatol. 1985;24:316-7.

Copyright by Darjani Abbas, et al. This is an open-access article distributed under the terms of the Creative Commons Attribution License, which permits unrestricted use, distribution, and reproduction in any medium, provided the original author and source are credited.

Source of Support: Nil, Conflict of Interest: None declared. 